\title{
Ionospheric topside models compared with experimental electron density profiles
}

\author{
Pierdavide Coïsson and Sandro M. Radicella \\ The Abdus Salam International Centre for Theoretical Physics (ICTP), Trieste, Italy
}

\begin{abstract}
Recently an increasing number of topside electron density profiles has been made available to the scientific community on the Internet. These data are important for ionospheric modeling purposes, since the experimental information on the electron density above the ionosphere maximum of ionization is very scarce. The present work compares NeQuick and IRI models with the topside electron density profiles available in the databases of the ISIS2, IK19 and Cosmos 1809 satellites. Experimental electron content from the $F 2$ peak up to satellite height and electron densities at fixed heights above the peak have been compared under a wide range of different conditions. The analysis performed points out the behavior of the models and the improvements needed to be assessed to have a better reproduction of the experimental results. NeQuick topside is a modified Epstein layer, with thickness parameter determined by an empirical relation. It appears that its performance is strongly affected by this parameter, indicating the need for improvements of its formulation. IRI topside is based on Booker's approach to consider two parts with constant height gradients. It appears that this formulation leads to an overestimation of the electron density in the upper part of the profiles, and overestimation of TEC.
\end{abstract}

Key words topside ionosphere - electron density models - topside soundings

\section{Introduction}

Information on the topside electron concentration distribution is not obtainable from groundbased measurements. In the past decades few satellites were equipped with ionosondes used for ionosphere sounding above the $F 2$ maximum (topside). Moreover, only a few percent of data recorded in the $60 \mathrm{~s}$ and $70 \mathrm{~s}$ were processed at that time due to the limited techniques available for automatic ionogram scaling. However, recently large amounts of newly scaled ionograms have been made available on-line (Bilitza et al., 2003). This kind of data is particularly important for ionospheric modeling purposes, since ionos-

Mailing address: Dr. Pierdavide Coïsson, The Abdus Salam International Centre for Theoretical Physics (ICTP), Strada Costiera 11,34014 Trieste, Italy; e-mail: coissonp@ictp.it pheric electron density models have their topside formulation based on old databases or different kind of measurements. Experimental vertical profiles are very important for model comparison, because they provide the spatial distribution of the electron concentration and they contain the information on $f o F 2$, which is generally used by models as an anchor point to describe vertical profiles. The present work analyzes the behavior of the NeQuick and IRI models, adopted by International Telecommunication Union, Radiocommunication sector (ITU-R) Recommendation P. 531-6 (now superseded by P. 531-7) (ITU, 2001), with respect to the topside electron density profiles available in the databases of the ISIS2, IK19 and Cosmos 1809 satellites. This work is part of the Italian degree thesis in physics thesis of one author (Coïsson, 2002).

\subsection{NeQuick model}

NeQuick model is an ionospheric electron density model, based on the original DGR «pro- 
filer» (Di Giovanni and Radicella, 1990). It is a quick-run model for trans-ionospheric applications that enables calculation of both vertical or slant electron density profile and TEC for any specified path (Hochegger et al., 2000; Radicella and Leitinger, 2001; Leitinger et al., 2002). Above $100 \mathrm{~km}$ and up to the $F 2$-layer peak this model uses a modified DGR profile formulation, which includes five semi-Epstein layers with modeled thickness parameters and is based on anchor points defined by $f_{O} E, f_{O} F 1$, foF 2 and $M(3000) F 2$ values. The topside is given by a semi-Epstein layer with a height dependent thickness parameter empirically determined.

The model has been adopted for assessment studies by the European Space Agency European Geostationary Navigation Overlay Service (EGNOS) project and more recently by ITU-R (ITU, 2001) as a suitable method for TEC modeling. The standard NeQuick source code is available at <http://www.itu.int/ITU-R/software/ study-groups/rsg3/databanks/ionosph>.

\subsection{IRI model}

The International Reference Ionosphere (IRI) is a well known and widely used empirical model of the ionosphere (Bilitza 1990, 2001). For a given location, date and time, IRI describes the electron concentration, electron temperature, ion temperature and ion composition in the altitude range from about $50 \mathrm{~km}$ to $2000 \mathrm{~km}$, as well as the TEC. IRI provides monthly averages in the non-auroral ionosphere for magnetically quiet conditions. It is periodically updated and has evolved over a number of years.

IRI model can be run on-line at $<$ http://nssdc.gsfc.nasa.gov/space/model/models/iri.html> or the source code can be retrieved via anonymous ftp from the NSSDC (National Space Science Data Center) site <ftp://nssdcftp.gsfc.nasa.gov/models $>$.

\section{Experimental data used}

The databases of topside electron density profiles from the International Satellite for Ionos- phere Studies 2 (ISIS2) (ftp://nssdcftp.gsfc.nasa.gov/spacecraft_data/isis/topside_sounder), Intercosmos 19 (IK19) and Cosmos 1809 satellites (http://antares.izmiran.rssi.ru/projects/IK19) were used for model comparisons. The betterquality experimental data were selected for model comparisons. The geographical distributions of the selected profiles are shown in fig. 1. The ISIS2 satellite was in a circular orbit at $1400 \mathrm{~km}$ height and available soundings were recorded from 1972 to 1983 ; the IK19 satellite was in an elliptical orbit between $500 \mathrm{~km}$ and $980 \mathrm{~km}$ height, with available data recorded in 1979 and 1980. Cosmos 1809 was in a circular orbit at 980 $\mathrm{km}$ height and data used were recorded during 1987.

The NeQuick and IRI models were used to compute modeled topside profiles corresponding to the experimental ones; the peak values ( $f o F 2$ and $h m F 2$ ) obtained from the topside experimental profiles were provided as input to the models, to focus the comparison on the shape of the topside profile. Other inputs given, which affect the modeled profile shape, are geographic location, universal time and solar activity, expressed by $R_{12}$. Figure 2 shows some example of experimental and modeled topside profiles for ISIS2 and IK19 satellites.

\section{Results}

Since there are no universally accepted criteria to compare an experimental profile with a modeled one, two quantities were considered in this work: Total Electron Content (TEC) difference from $h m F 2$ up to the satellite height; electron density ratio, $N_{\text {model }} / N_{\text {exp }}$, at a fixed height above the peak.

For statistical purposes, the data have been divided into homogeneous groups of: solar activity level, season of the year, modip and local time of the measurement. Median values and the upper and lower quartiles of the distributions in each sector were computed. Data recorded by different satellites were kept separated, but the results show consistent behavior.

In the statistical analysis performed the modified dip (modip) coordinate was used. Modip $\mu$ is defined by 

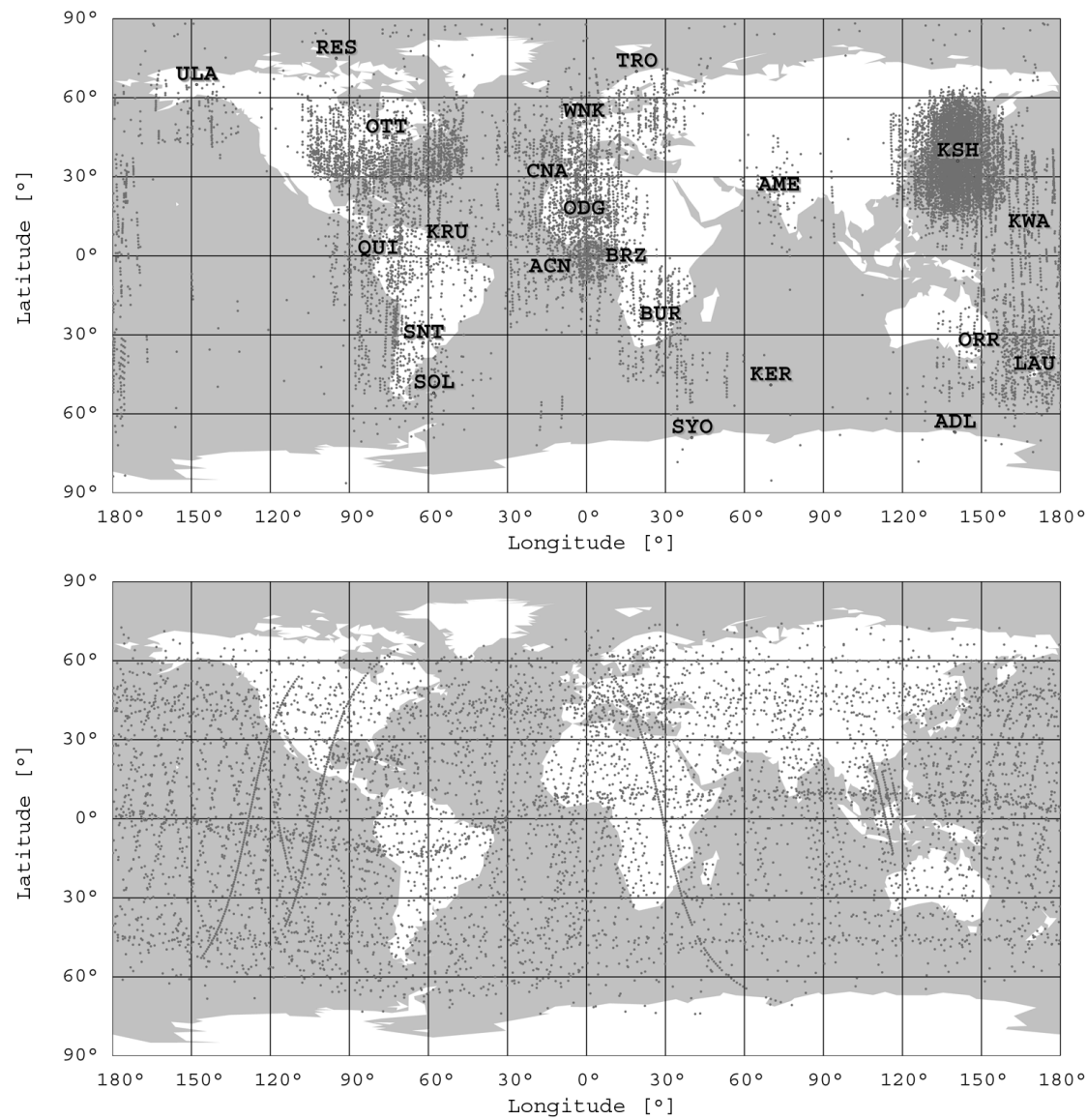

Fig. 1. Locations of the available profiles: (top panel) ISIS2 data, names indicate the telemetry stations used; (bottom panel) IK19 data.
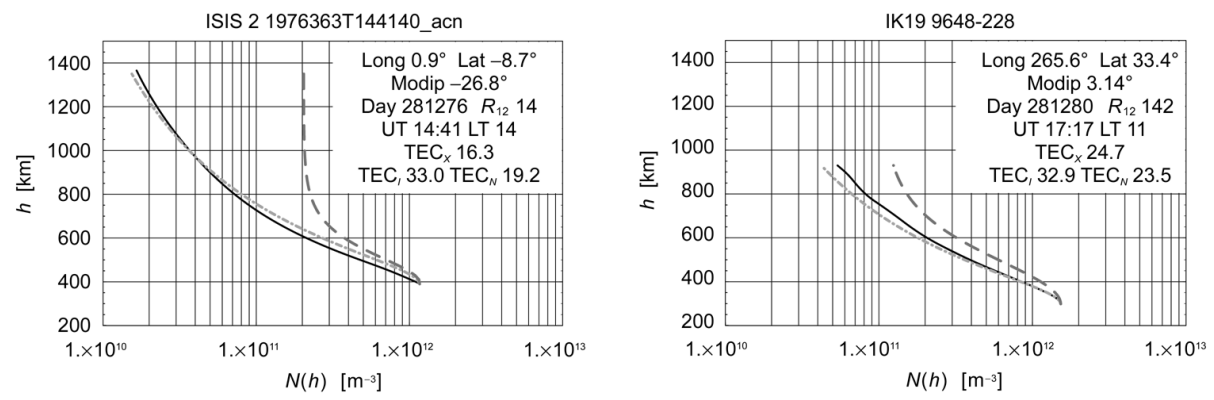

Fig. 2. Comparison between experimental profiles from ISIS2 and IK19 data and NeQuick and IRI models: experimental $(X)$ solid black, NeQuick $(N)$ dash-dotted light gray, IRI $(I)$ dashed dark gray. TEC in $10^{16} \mathrm{~m}^{-2}$. 


$$
\tan \mu=\frac{\psi}{(\cos \phi)^{1 / 2}}
$$

$\psi$ being the true magnetic dip in the ionosphere (usually at $300 \mathrm{~km}$ ) and $\phi$ the geographic latitude (Rawer, 1963). Data were divided into modip intervals to cover the different regions: equatorial region $\left(-5^{\circ}, 5^{\circ}\right)$, anomaly regions $\left(-35^{\circ},-5^{\circ}\right)$ and $\left(5^{\circ}, 35^{\circ}\right)$, mid-latitudes $\left(-60^{\circ},-35^{\circ}\right)$ and $\left(35^{\circ}, 60^{\circ}\right)$ and high-latitudes $\left(-90^{\circ},-60^{\circ}\right)$ and $\left(60^{\circ}, 90^{\circ}\right)$. Months were analyzed in three groups: first group from November to February, second group from May to August, third group March, April, September and October. Various local time intervals were considered: day from 09:00 to 17:00 LT, night from 21:00 to 05:00 LT, sunrise from 05:00 to 07:00 LT, sunset from 18:00 to 20:00 LT. For low solar activity $R_{12}<40$ was considered and for high solar activity $R_{12}>100$.

\subsection{TEC comparisons}

The percent differences between each modeled and experimental TEC value integrated from $h m F 2$ up to the satellite height were calculated and a statistical analysis performed as indicated in the previous section, the most important results are given below.

NeQuick model - Figure 3 shows a different behavior in the first and second group of months. During the first one it overestimates and underestimates the TEC anti-symmetrically with respect to the magnetic equator. During the second one it shows a symmetric behavior, with overestimation at low latitudes and better agreement at medium and high latitudes. These differences are associated with the different formulation of the NeQuick topside thickness parameter in the different months (Radicella and Zhang, 1995).

IRI model - Figure 4 shows that IRI tends to overestimate the topside TEC, with a behavior dependent on solar activity. While during low solar activity there is a greater overestimation at low latitudes and a good agreement at medium latitudes, for high solar activity IRI underestimates TEC in the equatorial region but strongly overestimates it at high latitudes. Day and night behaviors are similar in trend but there is better agreement at low latitudes.
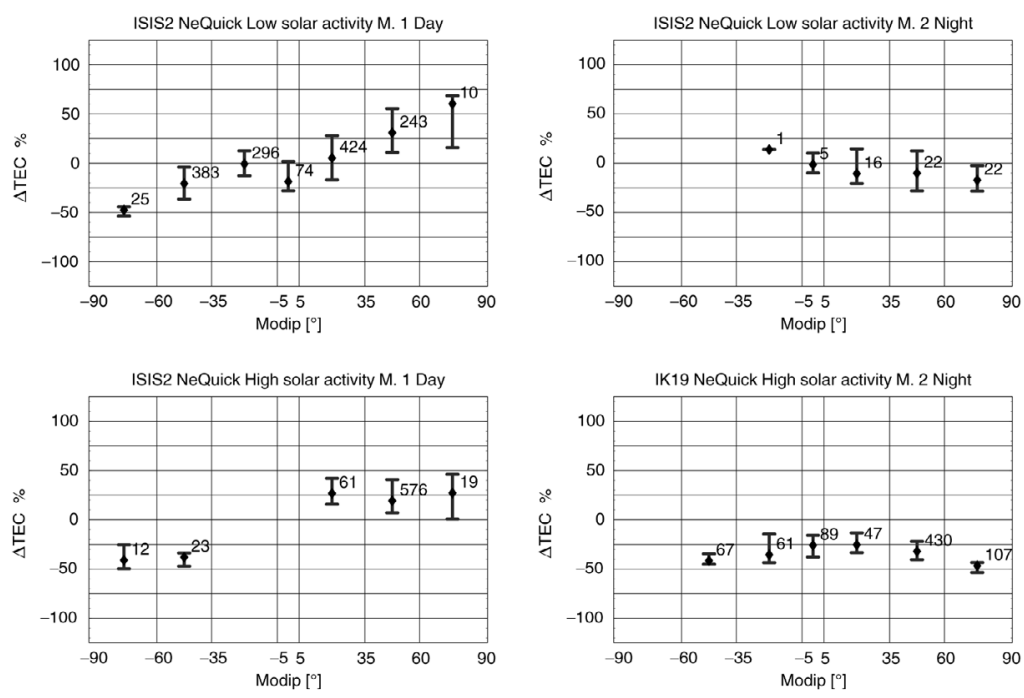

Fig. 3. Percent difference between experimental topside TEC and NeQuick topside TEC for months group 1 and 2. Numbers indicate the amount of data in each section. 

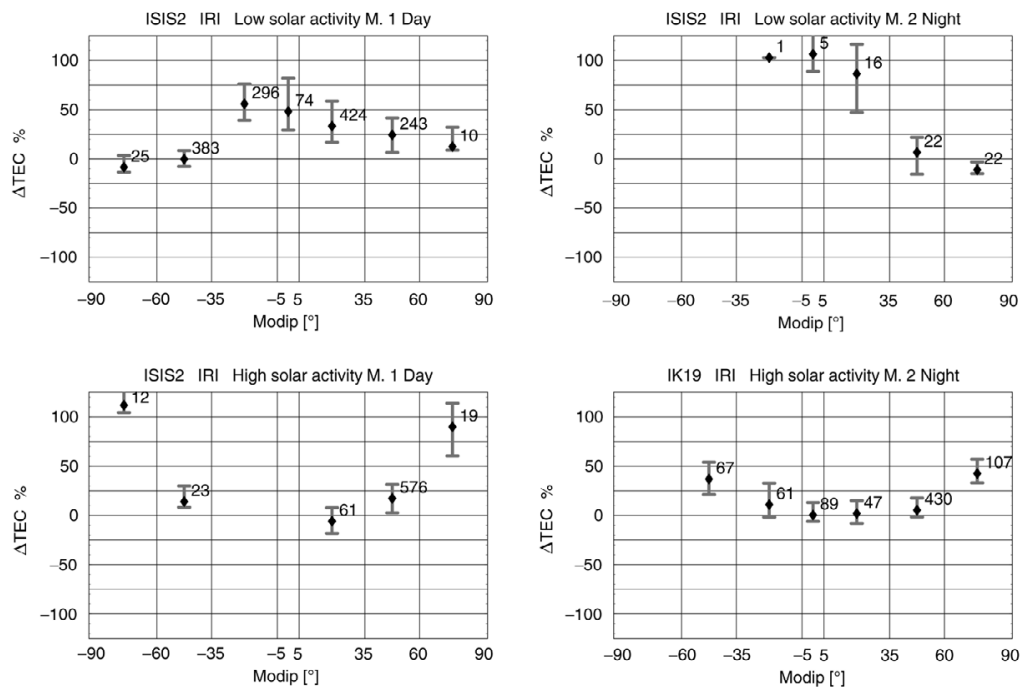

Fig. 4. Comparison of topside TEC, IRI model: percent difference for months groups 1 and 2. Numbers indicate the amount of data.
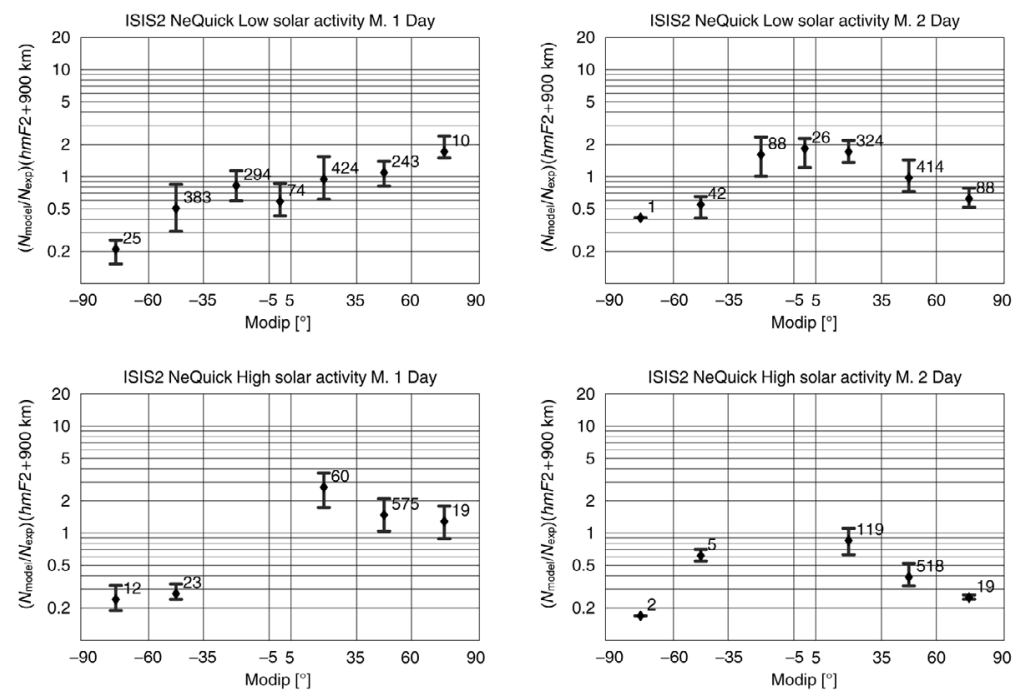

Fig. 5. Comparison of electron density, NeQuick model, at $900 \mathrm{~km}$ above $h m F 2$ for months groups 1 and 2. Numbers indicate the amount of data.

\subsection{Electron density comparisons}

The experimental and modeled electron densities $900 \mathrm{~km}$ above $h m F 2$ were compared. This height was reached only in the ISIS2 profiles, but it was chosen to assess models behavior in the upper part of the profile: the IRI model is used up to $2000 \mathrm{~km}$ and NeQuick to $20000 \mathrm{~km}$. 

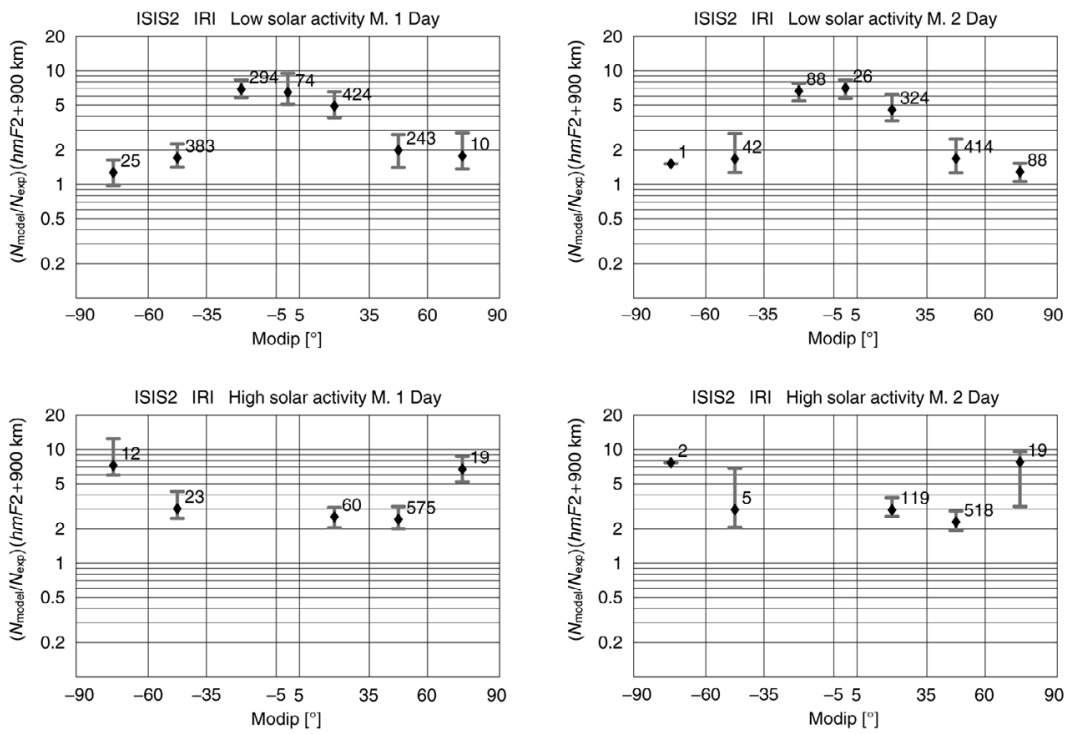

Fig. 6. Comparison of electron density, IRI model, $900 \mathrm{~km}$ above $h m F 2$ for months groups 1 and 2 . Numbers indicate the amount of data.

Thus it is important to understand how they are reliable in that region. Here results are shown only for local day time, because the number of data available for local night was not enough to provide good statistics in all regions. The small amount of night-time profiles confirms the results shown for day-time, the only relevant difference is indicated in the following description.

NeQuick model - Figure 5 shows a seasonal behavior similar to the one observed for topside TEC, with the electron density values contained between 0.2 and 3 times the experimental ones: little underestimation or overestimation. The behavior during day-time and night-time is similar, only for low solar activity can a difference be noticed at low modip, where the model goes from low underestimation to low overestimation and vice versa depending on month.

IRI model - Figure 6 demonstrates that IRI always overestimates the electron density; the median values are greater than 1.5 times the experimental ones and can reach up to 9 times. It shows a different modip behavior depending on solar activity level: during low solar activity the greater discrepancy is found at low modip, while for high solar activity the situation is reversed. At high latitudes for high solar activity cases have been found in which the IRI electron density does not decrease with height.

\section{Conclusions}

Comparisons have been made between 21000 topside electron density profiles from satellite measurements and those computed by the NeQuick and IRI models. Attention was focused on TEC and electron density at $900 \mathrm{~km}$ above the $F 2$ peak. The wide set of different experimental conditions has enabled assessment of many characteristics of each model.

The NeQuick topside is a modified Epstein layer. Its thickness parameter is governed by an empirical relation, with a dependence related to the month of the year. From this analysis it appears that the model performance is strongly affected by this parameter, indicating the need for an improvement of its formulation.

IRI is based on Booker's approach to model the topside by dividing it into two parts with 
constant height gradients. The gradients depend on latitude, solar activity and foF 2 . In the analysis carried out it appears that this formulation leads to overestimation of the electron density in the upper part of the profiles, and consequently to overestimation of TEC.

\section{REFERENCES}

BILITZA, D. (1990): International Reference Ionosphere 1990, NSSDC 90-22, Greenbelt, Maryland.

BiLitZA, D. (2001): International Reference Ionosphere 2000, Radio Sci., 36 (2), 261-275.

Bilitza D., B. Reinish, R. Benson, J. Grebowsky, N. PaPitashvili, X. Huang, W. Schar and K. Hills (2003): Online database of satellite sounder and insitu measurements covering two solar cycles, Adv. Space Res., 31 (3), 769-774.

CoÏsson, P. (2002): Uso di dati sperimentali per la convalida di modelli ionosferici applicati al disegno di servizi e sistemi satellitari, Thesis (Università degli Studi di Trieste).
Di GiovanNi, G. and S.M. Radicella (1990): An analytical model of the electron density profile in the ionosphere, Adv. Space Res., 10 (11), 27-30.

Hochegger, G., B. Nava, S.M. Radicella and R. LEITINGER (2000): A family of ionospheric models for different uses, Phys. Chem. Earth, 25 (4), 307-310.

ITU (2001): Ionospheric propagation data and prediction methods required for the design of satellite services and systems, Recommendation P.531-6, approved in 2001-02, managed by ITUR Study Group SG3 (version 6 superseded in 2004 by version 7).

LEITINGER, R., S.M. RADICELLA and B. NAVA (2002): Electron density models for assessment studies -new developments, Acta Geod. Geophys. Hung., 37 (2-3), 83-193.

RADICELLA, S.M. and R. LEITINGER (2001): The evolution of the DGR approach to model electron density profiles, Adv. Space Res., 27 (1), 35-40.

RAdiCELla, S.M. and M.L. ZHANG (1995): The improved DGR analytical model of electron density height profile and total electron content in the ionosphere, Ann. Geofis., XXXVIII (1), 35-41.

RAWER, K. (1963): Propagation of decameter waves (HFband), in Meteorological and Astronomical Influences on Radio Wave Propagation, edited by B. LANDMARK, (New York Academic Press), pp. 221-250. 